\title{
XIAN Automated Management and Nano-Protocol to Design Cross-Layer Metrics for Ad Hoc Networking*
}

\author{
Hervé Aïache, Vania Conan, Laure Lebrun, Jérémie Leguay, Stéphane Rousseau, \\ and Damien Thoumin \\ Thales Communications \\ 160, boulevard de Valmy, BP82 \\ 92704 Colombes Cedex, France \\ \{firstname.name\} @er. thalesgroup.com
}

\begin{abstract}
In the highly dynamic and unpredictable environment of MANETs, cross-layer design is receiving growing interest but lacks experimental validation tools. This paper presents XIAN (Cross-layer Interface for wireless Ad hoc Networks), a generic framework for experimenting cross-layer designs in Linux testbeds with 802.11 wireless cards using the MadWifi driver. XIAN can be used as a service by other layers or system components to access MAC/PHY configuration and performance information. It provides experimenters with an open framework to create automatically complex metrics from both local and neighbour node measurements. The defined and implemented software architecture introduces the XIAN Nano-protocol and its automated management. We exemplify their benefits through the implementation of the well-known ETX (Expected Transmission count) metric and we provide results from real experimentations.
\end{abstract}

\section{Introduction}

In Mobile Ad hoc Networks (MANETs [1]), nodes can be mobile and share one or more wireless channels without centralized control. In such dynamic and unpredictable distributed environments, traditional networking principles, such as the layer isolation of the OSI (Open Systems Interconnection) model, are challenged. Recent research studies have explored more flexible approaches to networking, called CrossLayer approaches, that revisit the classical IP stack design. The central idea of cross layering consists in allowing a more flexible exchange of status or control information between the different components of the communication system. With a better knowledge of available resources from different layers of the ad hoc network stack, the system is expected to be more reactive to the wireless environment and responsive to quality requirements of applicative-oriented elements.

When compared to the usual OSI reference model, existing cross-layer solutions span a wide spectrum of options: some advocate global exchange of information between components (e.g. Conti et al. [2]), others prefer to limit them to adjacent layers (e.g. Kawadia et al. [3]), depending on how they impact or differ from this reference

* This work has been partially supported by the European IST FP6 CHORIST project and the French RNRT Airnet project. 
model. In any case, cross-layering calls for software architectures and implementations that support a more flexible sharing of information and status reports between the processes and functional modules of the communication system. Experimenting with cross layer design for MANETs remains difficult, most ad hoc testbeds making use of 802.11 cards which lack appropriate API support.

This paper builds upon our earlier work [4] to define XIAN (Cross-layer Interface for wireless Ad hoc Networks) which consists in a framework that facilitates crosslayer integrations and experimentations by easing the access to information from MAC/PHY layers. The ultimate goal of XIAN is to encourage and facilitate crosslayer studies and experimentations over MANET testbeds. It has been implemented and is available in open source [7] for Linux over the MadWifi 802.11 driver [5]. This paper presents a major extension to the earlier basic framework by supporting the implementation of complex cross-layer metrics that can combine both local information and measurements obtained from the node's neighbours by the XIAN Nanoprotocol. We exemplify its use with the implementation of the ETX (Expected Transmission Count) metric proposed by De Couto et al. [6] and we provide experimental demonstration of potential benefits.

The remainder of the paper is structured as follows. Section 2 presents the XIAN approach and software architecture. Section 3 describes the XIAN programming interfaces and examples of accessible metrics. Section 4 and Section 5 present the use and experimental benefits of the proposed XIAN extension for the implementation of ETX. Section 6 concludes the paper, discussing directions for future work.

\section{XIAN: Cross-Layer Interface for Wireless Ad hoc Networks}

This section introduces the overall XIAN framework. We first present the Linux based software architecture. Then we show how to integrate new complex metrics to extend and customize the framework. Finally we describe the XIAN Nano-protocol, integrated at the MAC layer, that automatically handles metric exchanges between neighbour nodes.

\subsection{XIAN Basic Components for Cross-Layer Exchanges}

MAC/PHY state information, such as number of transmission retries or number of transmitted frames with CTS enabled, is available at driver level and a large part of this information may be of interest to higher layers. To support flexible cross-layer access to this information XIAN implements a software architecture composed of three main components:

- The Kernel Space XIAN Interface (KSI) is dedicated to kernel space components (e.g. TCP or UDP implementations) and implemented as a Linux kernel module. It interacts directly with the MadWifi driver to retrieve its internal states or statistics or with the XIAN components involved in the definition of complex metrics (see section 2.2). 
- $\quad$ The User Space XIAN Interface (USI) mimics the KSI but at user space level. This API is implemented as an ordinary C library in order to facilitate its integration with user space programs (e.g. routing daemons or applications).

- The XIAN Information Transport Module (ITM) allows to pass information and statistics from the kernel space to the user space, by connecting the two previous XIAN APIs. This module is implemented in this version of XIAN as a special character device.

In addition, a complementary component, called the XIAN User Space Extended Interface (or USEI), provides simple means for experimenters to perform additional processing of raw measurements, such as averaging, metric combination or notification of significant changes. Figure 1 illustrates how the components interact and how internal driver/MAC states or metrics are provided to other Linux system components.

From the developer's point of view, the two XIAN APIs (i.e. KSI and USI) are identical. The information exchanged through the ITM and accessible via the USI and the KSI are of two kinds: (1) basic metrics extracted from specific structures/states

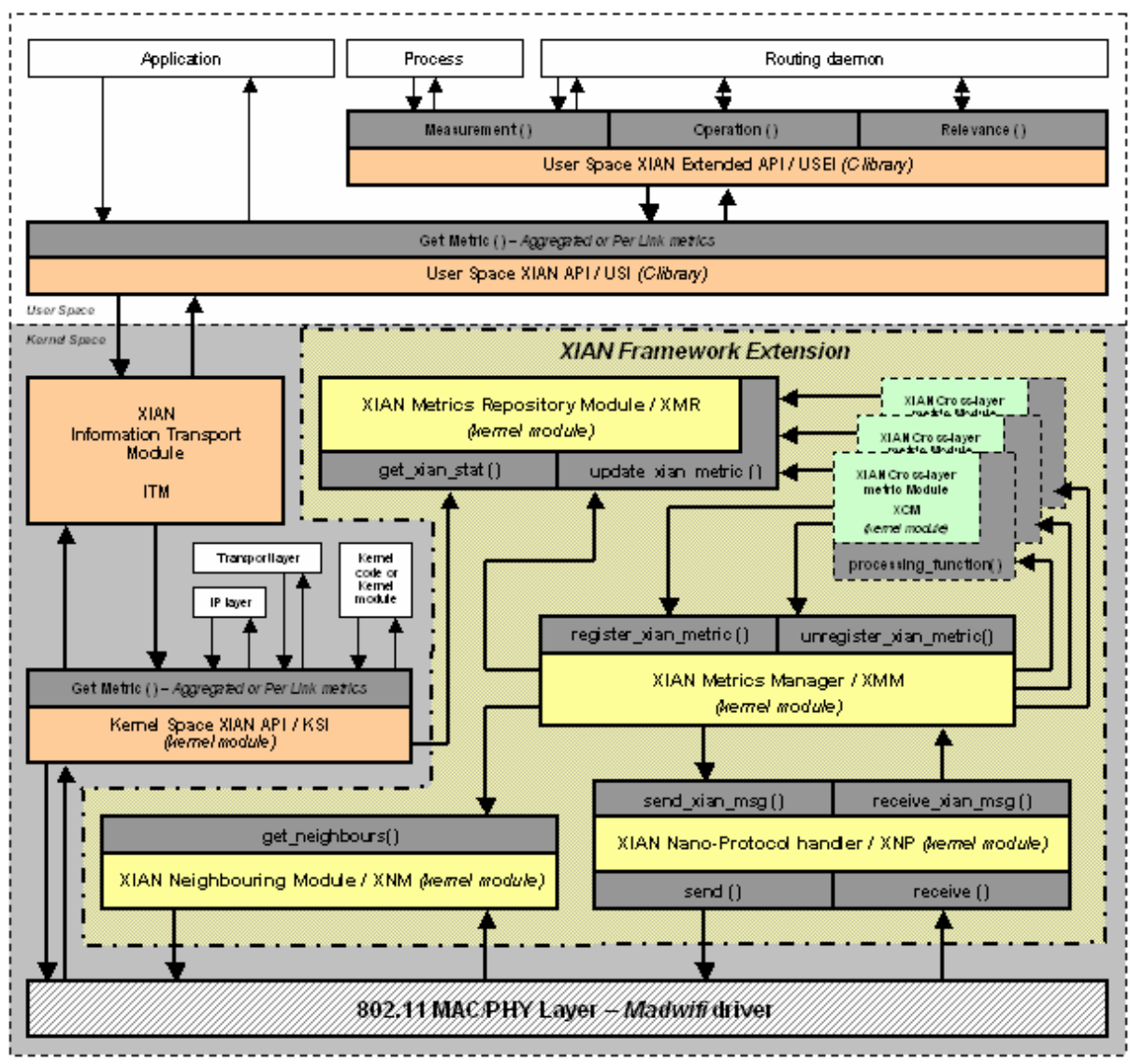

Fig. 1. XIAN Framework software architecture 
maintained by the MadWifi driver, and (2) new metrics integrated to the framework thanks to the software components described in the following section.

\subsection{Extension to Integrate and Manage Complex Cross-Layer Metrics}

XIAN offers mechanisms implementing bidirectional exchanges of metrics among neighbouring nodes (such as those classically needed by $[6,9,10,11]$ ). This makes possible the creation and the integration within the XIAN framework of metrics that require information from neighbouring nodes (e.g. the number of packets that have been received by a neighbour). Implementing a new metric involves the definition of the metric calculation formula, i.e., it defines how to combine readings from several individual PHY/MAC layer metric values to obtain a new compound one. Obtaining the individual values involves configuring the XIAN framework, by setting which metrics to read from the node's neighbours and at which frequency. The implementation of this major extension to XIAN is available in the new release on the official project web site [7].

As shown Figure 1, four software components, all implemented as Linux kernel modules, support the integration of such complex metrics within the XIAN framework:

- The XIAN Metric Manager (XMM), in charge of the registration and unregistration of the new cross-layer metrics instantiated as XIAN Cross-layer Metric modules (XCMs - An XCM mainly implements the metric calculation formula and configures the protocol exchanges involved in the calculation of the metric in terms of type of information and frequency - see section 3.2).

- The XIAN Nano-Protocol handler (XNP), which handles the XIAN Nanoprotocol messages containing the values of the metrics exchanged between neighbouring nodes. The XNP is implemented between the MadWifi driver and the Linux kernel implementation of the IP layer. Its role is to extract the XIAN Nano-protocol messages from in-coming 802.11 frames and to create and send out-going XIAN Nano-protocol messages to the desired neighbours.

- The XIAN Metrics Repository (XMR), which is responsible of recording the calculated values of the new cross-layer metrics introduced within the XIAN framework. Once recorded by the XMR, the metric values become accessible (via the KSI or the USI - see section 1.1) to other operating system components, along with all MadWifi driver information reported by default by XIAN.

- $\quad$ The XIAN Neighbouring Manager (XNM), which detects neighbour nodes and triggers updates necessary to automate XIAN Nano-protocol message exchanges.

To enable the exchanges of metrics between neighbouring nodes, we propose a simple MAC layer-oriented protocol, called the XIAN Nano-protocol. It is described in the following section.

\subsection{The XIAN Nano-Protocol}

The XIAN Nano-protocol is responsible of exchanging the XIAN Nano-protocol messages. Figure 2 gives an example of such a message. It contains a XIAN Nanoprotocol Metrics Reports made up of (1) a header, which mainly indicates information 
about the report object itself and (2) a payload, which can embed several XIAN Nanoprotocol Metric Objects that provides metric values and associated meta information. Note that XIAN Nano-protocol messages are encapsulated into unicast or broadcast 802.11 data frames.

The XIAN Nano-protocol Metrics Report is composed of the following fields:

- Version indicates the used version of the XIAN Nano-Protocol.

- Sequence is the identifier of the XIAN Nano-protocol Metrics Report.

- Length indicates the number of XIAN Nano-protocol Metric Objects contained in the XIAN Nano-protocol Metrics Reports.

- Payload contains a set of XIAN Nano-protocol Metric Objects.

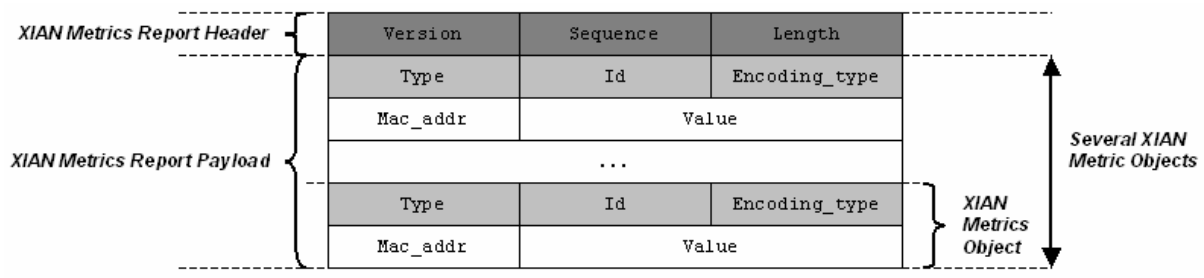

Fig. 2. Metrics Report format of the XIAN Nano-protocol

A XIAN Nano-protocol Metric Object is composed of the following fields:

- Type indicates the identifier associated to a metric.

- Id identifies a reference to the associated XCM (see section 3.2).

- Encoding_type indicates how the metric value is encoded (e.g. integer or float).

- Mac_addr indicates the MAC address to which the metric value belongs to.

- Value contains the effective numerical value of the metric.

Moreover, in order to optimize the number of messages sent to a given neighbour node, note that the XIAN Nano-Protocol handler (XNP) is able to aggregate several XIAN Cross-Layer Metrics Object of different types in a single XIAN Metrics Report.

\section{XIAN Programming Interfaces}

The previous section described the main building blocks of the XIAN architecture. This section details the programming interfaces offered by XIAN to implement view to access, refine, design and integrate MAC-oriented cross-layer metrics.

\subsection{Accessing MAC-Oriented Basic Metrics}

XIAN eases the access to a large set of basic metrics offered by the MadWifi driver. The most important ones can be divided into two groups:

- Global metrics, similar to counters, this kind of metrics provides global statuses on the use of the 802.11 network interface. The reported information can be: 
Note that with such a design, experimenters do not have to take care about the XIAN Nano-protocol exchanges, but just have to configure its behaviour, meaning: the frequency of the metrics reports (i.e. the parameter called freq), what are the nodes involved in the calculation of the metric (i.e. the parameter mac) and the formula to calculate the metric (i.e. the processing function $\mathrm{pf}$ ).

In addition to the function register_id(), the XMM proposes another complementary interface which allows to un-register an XCM:

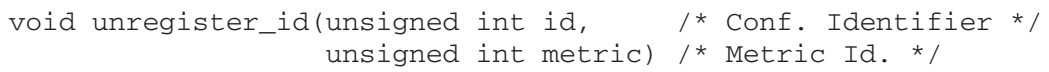

This function un-register the metric identified within XIAN by its reference metric and its associated XCM (or configuration) identifier id.

Therefore, as mentioned, a typical XCM implementation looks like to a classical Linux kernel module with specific calls to the functions register_id ( ) and unregister_id ( ). In this way, a typical XCM would implement the following functions:

- An initialisation function, as for Linux kernel modules implementation, which is necessary to load the XCM into the Linux kernel and to register the new metric (or several) within the XIAN framework thanks to the XMM's function called register_id().

- A processing function, which is specific to XIAN and enables the reception of XIAN Nano-protocol Metric Objects in view to extract the metrics values and to apply the associated formula before updating the metric values through the XMR, the metrics repository of the XIAN framework.

- A cleanup function, as for Linux kernel modules implementation, which is important to unload properly the XCM from the Linux kernel and to unregister the given cross-layer metric (or several) from XIAN thanks to the XMM's function called unregister_id( ) .

Since the processing function performs operations on the metrics exchanged through the XIAN Nano-protocol messages, it follows a specific prototype:

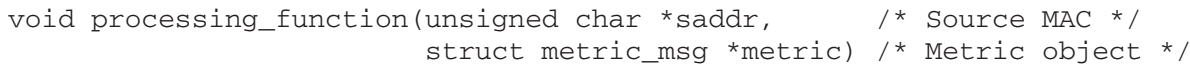

Thanks to this function, once the XCM is inserted inside the Linux kernel and registered within the XIAN framework, the XMM is able to pass the received XIAN Cross-Layer Metric Object in metric to the right XCM. Moreover, note that the $\mathrm{XMM}$ provides an additional information to the given XCM: the MAC address of the source node (indicated by the variable saddr), which sent the received XIAN CrossLayer Metric Object (i.e. contained in metric).

\subsection{XIAN User Space Extended Interface}

Several metrics may have to be further combined or refined in order to be meaningful or at least more useful for specific system components. For example, the number of transmitted MAC frames (in bytes) would not be an interesting metric if no timecorrelation is introduced to reflect how this metric evolves during the life-time of the wireless communication system. In other cases, the average value of a given metric is 
more meaningful than an instantaneous measurement. Therefore, XIAN complements the interfaces introduced in Section 3.1 and 3.2 with another API, available in userspace, called the User Space XIAN Extended Interface (USEI), which provides:

- Measurement functions, which compute metrics resulting from the combination of several elementary metrics taken directly via the USI or from the refinement of an elementary metric (e.g. average values).

- Operation functions, which implement the corresponding mathematical operator required by the new defined calculated metrics (e.g. min or max functions).

- Relevance functions, which implement the corresponding comparator indicating if a significant difference occurs between two calculated values (typically between the previous and the new ones).

The USEI has been implemented as a usual C library to facilitate its use and integration with user space processes. Based on the description of the XIAN architecture and programming interfaces, the following section exemplifies their use with the implementation of the ETX (Expected Transmission Count [6]) metric as a XIAN Crosslayer Metric module (XCM) available as an example of complex metric in the new release of XIAN.

\section{Implementation of a Complex Metric within XIAN}

Several cross-layer metrics have been proposed in the literature. De Couto et al. $[6,8]$ have proposed the Expected Transmission count (ETX) which measures the bidirectional packet loss ratio of link. Awerbuch et al. [9] have introduced the Medium Time Metric (MTM) that selects high throughput paths. Déziel et al. [10] have defined the available bandwidth. Iannone et al. [11] have combined the packet success rate, the interference level, and the physical bit rate.

In this section, we specifically illustrate how the new set of XIAN functionalities presented in this paper enable experimenters to easily implement the ETX metric. First, we recall the definition of this metric. Then, we show how to implement this metric within the XIAN framework.

\subsection{The Expected Transmission Count (ETX)}

The Expected Transmission count (ETX) calculates the expected total number of packet transmissions (including retransmissions) required to successfully deliver a packet to the ultimate destination. ETX predicts the number of transmissions required using per-link measurements of packet loss ratios in both directions of wireless links.

The ETX value for a route is the sum of the link metrics. ETX is a combination of two measurements: (1) the forward delivery ratio, called $D_{f}$, which is the measured probability that data packets successfully arrive at the recipient, and (2) the reverse delivery ratio, $D_{r}$, which is the probability that ACKs packets are successfully received. The probability that a transmission is successfully received and acknowledged is $D_{f} \times D_{r}$. Then, ETX is expressed as follows: 


$$
\operatorname{ETX}\left(\text { link) }=1 /\left(\begin{array}{lll}
D_{f} & \times & D_{x}
\end{array}\right) .\right.
$$

The main issue when it comes to ETX implementation is that $D_{f}$ and $D_{r}$ are not directly available locally. The only solution is to exchange delivery ratios among neighbours. These delivery ratio can be determined in various manners: (1) by looking at statistics reported in a per-neighbour fashion by XIAN (e.g., number of data transmission trials, number successful data transmissions), (2) by comparing the number of broadcast packets that have been received from neighbours to those that should have been received, assuming that nodes send broadcast messages every $T$ milliseconds.

In this work, to demonstrate how ETX can be implemented as a XIAN Cross-layer Metric modules, nodes exchange their delivery ratios for all their out-going links using XIAN Nano-protocol messages. They also take advantage of these message exchanges to calculate these delivery ratios using the solution (2) explained in the previous paragraph.

\subsection{ETX Implementation in XIAN}

ETX has been implemented within the XIAN framework as an XCM, called ETX_XCM. At the load of the ETX_XCM, we configure the XNM to send every Tmilliseconds the metric $D_{r}$ in a broadcast message. Thus we create a new configuration identifier and a new metric code for it. The parameter $\mathrm{pf}$ given to the function register_id ( ) is a function called at each reception of the metric ETX, which has the following prototype:

processing_ETX (unsigned char*saddr, struct metric msg *metric).

The broadcast message contains the values of $D_{r}$ for the list of MAC addresses provided by the function insert_mac_to_broadcast () which is used for each received broadcast message when the MAC address is not present. At the reception of those messages, the ETX_XCM stores the $D_{r}$ metric's value thanks to the XMR by using its interface: update_xian_stat (). Then, ETX_XCM increments a counter and records it in the XMR. Thanks to $D_{r}$ and $D_{f}$, we can calculate ETX. The calculated ETX values are stored within the XMR in order to provide the metric and the possibility to estimate the quality of a link.

In parallel, a thread is wake up every $W$ milliseconds to recover the counter and to calculate the $D_{f}$, thanks to the counter and the expected number of received messages during $W$ milliseconds. $D_{f}$ is then recorded within the XMR and the counter reset to zero. At last, ETX is recalculated with the new value of $D_{f}$ and updated within the XMR.

Note that, since the Linux kernel does not manage float values, all the metrics within the kernel space are under the format struct xian_float and accessible by the function struct xian_float get_xian_stat(), added to the KSI. In the user space, the results are available under the format float with the similar function float get_xian_stat(), offered by the USI. Moreover, to perform operations on struct xian_float, the addition, the multiplication and the division are available as functions provided by XIAN. 
Based on this implementation of ETX, the following section explains how the ETX_XCM has been used to perform measurements over a real 802.11 ad hoc testbed.

\section{Experimental Testbed and Results}

To illustrate the benefit of XIAN to facilitate cross-layering experiments, we present experimental measurements oriented on QoS routing and performed with the $E T X \_X C M$, the implementation of ETX with the XIAN APIs.

The testbed we setup was composed of 3 machines equipped with Cisco Aironet Wi-Fi cards equipped by the Atheros chipset and configured to use the $802.11 \mathrm{~b}$ standard at the bit rate of $11 \mathrm{Mbits} / \mathrm{s}$ in ad hoc mode (without RTS/CTS). On each machine, we used iperf [12] to generate TCP and UDP traffic and the STAF/STAX [13] framework to automate the experiment runs. We also use a simple program that logs, for each link, the metrics accessed through XIAN. We logged each of the following metrics every $\delta$ seconds:

- The $\boldsymbol{R S S I}$ (Relative Signal Strength Indicator): the wide-band received power within the used channel;

- The throughput: the sum of total data bytes received and sent at the MAC layer within the last $\delta$ seconds;

- The $\boldsymbol{E T X}$ metric measured over 10 beacons (XIAN Nano-protocol messages) sent every 200 milliseconds.

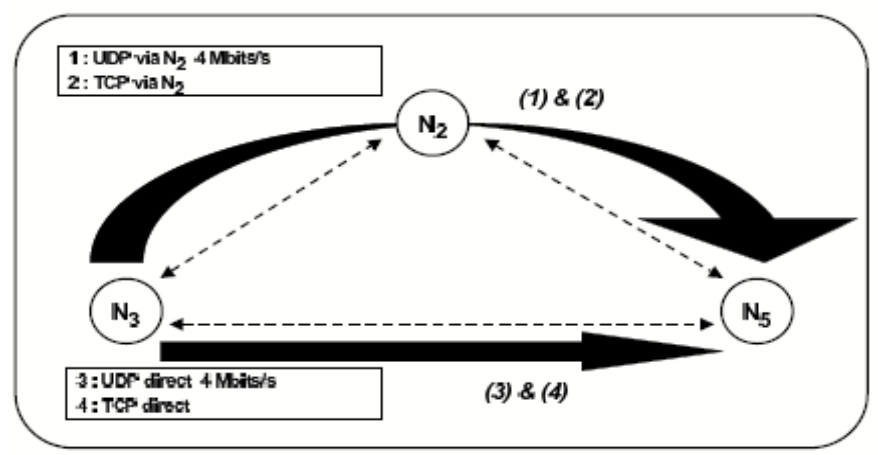

(a) Network topology

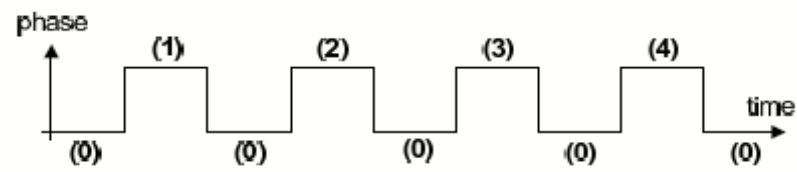

(b) Traffic pattern

Fig. 3. Experimental setup and traffic generation 
Figure 3 presents the triangle network topology that we considered and the different flows that we have generated. The link between the machine $\mathrm{N}_{2}$ and $\mathrm{N}_{5}$ is significantly longer than the other ones. The traffic generation sequence used (depicted in Figure 3(b)) is composed of the following phases each lasting 24 seconds:

- $\quad$ Phase (0): no traffic.

- $\quad$ Phase (1): UDP traffic between $\mathrm{N}_{3}$ and $\mathrm{N}_{5}$ via $\mathrm{N}_{2}$ at 4 Mbits/s.

- $\quad$ Phase (2): TCP traffic between $\mathrm{N}_{3}$ and $\mathrm{N}_{5}$ via $\mathrm{N}_{2}$.

- Phase (3): UDP traffic between $\mathrm{N}_{3}$ and $\mathrm{N}_{5}$ at 4 Mbits/s.

- Phase (4): TCP traffic between $\mathrm{N}_{3}$ and $\mathrm{N}_{5}$.

Figure 4 presents the measurements that we have performed. More specifically, Figure 4(b) and Figure 4(e) present the throughput respectively for links $\mathrm{N}_{3} \rightarrow \mathrm{N}_{2}$ and $\mathrm{N}_{3} \rightarrow \mathrm{N}_{5}$. We can see that when UDP and TCP flows issued from $\mathrm{N}_{3}$ are passing via $\mathrm{N}_{2}$ to reach $\mathrm{N}_{5}$, high throughput are achieved with average values equal respectively to 3.4 Mbits/s (saturation is achieved) and $2.4 \mathrm{Mbits} / \mathrm{s}$. Whereas when UDP and TCP flows issued from $\mathrm{N}_{3}$ are passing directly over the link $\mathrm{N}_{3} \rightarrow \mathrm{N}_{5}$, the average values are equal respectively to $0.1 \mathrm{Mbits} / \mathrm{s}$ and $0.1 \mathrm{Mbits} / \mathrm{s}$. These results show that in our case, routing decisions based on hop-count can fail. Cross-layer metrics are then required to achieve acceptable performances.

Figure 4(a) and Figure 4(d) look at RSSI values respectively for links $\mathrm{N}_{3} \rightarrow \mathrm{N}_{2}$ and $\mathrm{N}_{3} \rightarrow \mathrm{N}_{5}$. We can see that due to the higher length of the link $\mathrm{N}_{3} \rightarrow \mathrm{N}_{5}$, RSSI values are lower on this link than those or the link $\mathrm{N}_{3} \rightarrow \mathrm{N}_{2}$ with average values equal respectively to $12.5 \mathrm{Db}$ and $36.1 \mathrm{Db}$. An average RSSI of $33.4 \mathrm{Db}$ have been observed for the link $\mathrm{N}_{2} \rightarrow \mathrm{N}_{5}$. In our case, we could have used this metric to make efficient routing
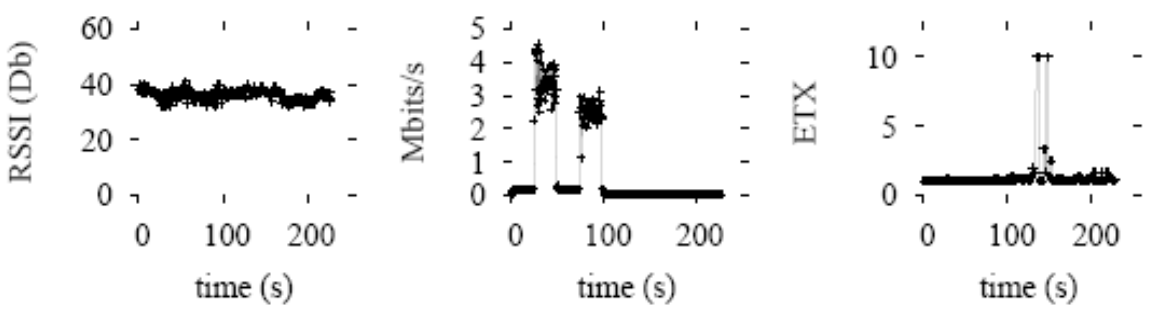

(a) RSSI $N_{3} \rightarrow N_{2}$

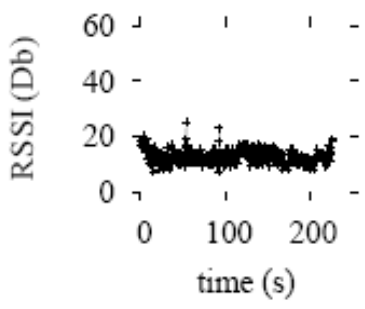

(b) Throughput $N_{3} \rightarrow N_{2}$

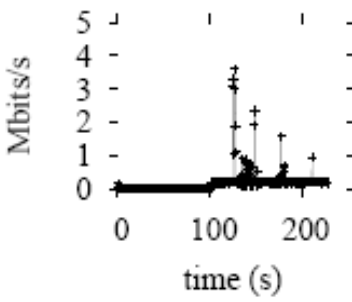

(c) $\operatorname{ETX~} N_{3} \rightarrow N_{2}$

(d) RSSI $N_{3} \rightarrow N_{5}$

(e) Throughput $N_{3} \rightarrow N_{5}$

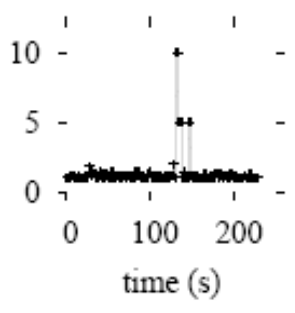

(f) $\operatorname{ETX} N_{3} \rightarrow N_{5}$

Fig. 4. Metric values measured with XIAN 
decisions. However, the RSSI does not capture information regarding the congestion and contention levels at MAC layer and thus may not work in larger and more realistic conditions.

Figure 4(c) and Figure 4(f) present the ETX values respectively for links $\mathrm{N}_{3} \rightarrow \mathrm{N}_{2}$ and $\mathrm{N}_{3} \rightarrow \mathrm{N}_{5}$. We can see that the link $\mathrm{N}_{3} \rightarrow \mathrm{N}_{5}$ plots ETX values continuously higher than the one of the link $N_{3} \rightarrow N_{2}$ (see especially values for the different phases (0) ) which denotes the fact that ETX captures the poor quality of links. In phases (3), as the UDP traffic is totally not able to be transferred over the link, node $\mathrm{N}_{3}$ runs out of buffer memory. This results in a large amount of drops for the beacons used in ETX computation and thus to high values of the metric (this impact can also be seen for the ETX value of link $\mathrm{N}_{3} \rightarrow \mathrm{N}_{2}$ ). Some of the loses of beacons might have also caused by collisions over the wireless medium. We have seen here that ETX captures two crucial factors: (1) the weakness of links due to low RSSI values and (2) the local and neighbouring traffic load.

This experiment illustrates how the ETX metric, implemented within the XIAN Framework through automated XIAN Nano-protocol exchanges, introduced in this paper, allows to make efficient QoS routing decisions.

Note that the purpose of this work was to show how to implement the original ETX specifications in XIAN. With XIAN it is moreover possible to explore variants of this protocol, for example by monitoring more exactly the real traffic to estimate transmission count in place of the dedicated ETX probes, thus taking into account the packet size distribution.

\section{Conclusions and Future Work}

This paper presented XIAN a cross-layer interface implementation specialized in the building of experimental setups for validating a large variety of use cases of IEEE 802.11 cross-layering and its extensions to support complex user-defined metrics. The extension proposed in this paper turns XIAN into an extensible framework dedicated to the creation of complex cross-layer metrics. Moreover, the decomposition of crosslayer metric definitions in metric calculation formula and protocol exchanges configuration, translated into software components, facilitates their design and their integration within the XIAN framework.

It is thus possible to define and to implement cross-layer metrics as new components in XIAN that can use both local and neighbouring measurements transparently exchanged with the XIAN Nano-protocol. We exemplified its use with the implementation of the ETX (Expected Transmission Count) metric and we provided experimental demonstration of its potential benefits. Finally, we released our code that can be downloaded from [7].

Future work along these lines would include the development of interfaces working in a publish/subscribe manner. This kind of interface may improve further the integration of the MAC and routing layers as it would allow, for instance, reporting of link up and link down events and help the system react more quickly to topology changes. Finally, one could wish to extend the generic APIs to support other chipsets than Atheros in the spirit of the Wireless Tools. 


\section{References}

1. Corson, S.: RFC 2501, Mobile ad hoc networking (MANET): Routing protocol performance issues and evaluation considerations. In: IETF (January 1999)

2. Conti, M., Maselli, G., Turi, G., Giordano, S.: Cross-layering in mobile ad hoc network design. IEEE Computer, 48-51 (February 2004)

3. Kawadia, V., Kumar, P.R.: A cautionary perspective on cross layer design. IEEE Wireless Communication Magazine (July 2003)

4. Aïache, H., Conan, V., Leguay, J., Levy, M.: XIAN: Cross-layer interface for wireless ad hoc networks. In: Proc. Med-Hoc-Net (2006)

5. MadWifi, http://www.madwifi.org

6. De Couto, D.S.J., Aguayo, D., Bicket, J., Morris, R.: A high-throughput path metric for multi-hop wireless routing. In: Proc. MobiCom. (2003)

7. XIAN., http://sourceforge.net/projects/xian/

8. De Couto, D.S.J., Aguayo, D., Chambers, B.A., Morris, R.: Performance of multi-hop wireless networks: Shortest path is not enough. In: Proc. HotNets, ACM SIGCOMM (2002)

9. Awerbuch, B., Holmer, D., Rubens, H.: High throughput route selection in multi-rate ad hoc wireless networks. In: Proc. WONS. (2004)

10. Déziel, M., Lamont, L.: Implementation of an IEEE 802.11 link available bandwidth algorithm to allow cross-layering. In: Proc. WiMob. (2005)

11. Iannone, L., Khalili, R., Salamatian, K., Fdida, S.: Cross-layer routing in wireless mesh networks. In: Proc. ISWCS. (2004)

12. Iperf, http://dast.nlanr.net/Projects/Iperf/

13. STAF: Software Testing Automation Framework, http://staf.sourceforge.net 